\title{
How "healthy" should children be when selecting reference samples for spirometry?
}

Sooky Lum¹, Vassiliki Bountziouka', Samatha Sonnappa ${ }^{1,2}$, Tim J. Cole $^{3}$, Rachel Bonner ${ }^{1}$ and Janet Stocks ${ }^{1}$

Affiliations:

${ }_{1}^{1}$ Respiratory, Critical Care and Anaesthesia section (Portex Unit), University College London, Institute of Child Health, London, UK.

${ }^{2}$ Institute of Global Health, University College London, Institute of Child Health, London, UK.

${ }^{3}$ Population, Policy and Practice Programme, University College London, Institute of Child Health, London, UK.

Correspondence: Sooky Lum, Respiratory, Critical Care and Anaesthesia section (Portex Unit), University College London, Institute of Child Health, 30 Guilford Street, London, WC1N 1EH, UK. E-mail: s.lumaucl.ac.uk

ABSTRACT How "healthy" do children need to be when selecting reference samples for spirometry?

Anthropometry and spirometry were measured in an unselected, multi-ethnic population of school children aged 5-11 years in London, UK, with follow-up assessments 12 months later. Parents provided information on children's birth data and health status. Forced expiratory volume in $1 \mathrm{~s}$ (FEV1) and forced vital capacity (FVC) were adjusted for sex, age, height and ethnicity using the 2012 Global Lungs Initiative equations, and the effects of potential exclusion criteria on the z-score distributions were examined.

After exclusions for current and chronic lung disease, acceptable data were available for 1901 children on 2767 occasions. Healthy children were defined as those without prior asthma or hospitalisation for respiratory problems, who were born at full-term with a birthweight $\geqslant 2.5 \mathrm{~kg}$ and who were asymptomatic at testing. Mean $\pm \mathrm{SD}$ Z-scores for FEV1 and FVC approximated $0 \pm 1$, indicating the 2012 Global Lungs Initiative equations were appropriate for this healthy population. However, if children born preterm or with low birthweight, children with prior asthma or children mildly symptomatic at testing were included in the reference, overall results were similar to those for healthy children, while increasing the sample size by $25 \%$.

With the exception of clear-cut factors, such as current and chronic respiratory disease, paediatric reference samples for spirometry can be relatively inclusive and hence more generalisable to the target population.

@ERSpublications

Population samples for children's lung function can be relatively inclusive http://ow.ly/I0Jkw 


\section{Introduction}

The inclusion and exclusion criteria applied to subjects in population-based studies of lung function vary according to the underlying question and study design [1]. Excluding subjects with prior potentially adverse exposures may be appropriate when establishing normative data for reference equations [2, 3], but less so in studies exploring the early determinants of lung function during childhood [4]. Furthermore, when collecting data in schools it may be more efficient to include all children and subsequently exclude some, rather than exclude children on "health" grounds at the outset, which may cause embarrassment and upset. Similarly, although paediatric research studies often exclude lung function measurements within 3 weeks [5] or 6 weeks [2] of upper respiratory infections, children frequently suffer from such symptoms and their impact of such symptoms on the results is unclear. Reassessing the child when they are symptom-free is not as easy for school-based studies as laboratory studies [5].

The aim of this study was to examine the extent to which exclusions due to current upper respiratory symptoms or a history of potential adverse events, such as low birthweight, preterm birth or prior wheezing/asthma, impact on the distribution of spirometric z-scores in the context of a large population-based study.

\section{Methods}

The Size and Lung function In Children (SLIC) study was designed to explore ethnic differences in lung function and body physique in a multi-ethnic population of school children aged 5-11 years recruited from 14 primary schools in London, UK (2010-2013) (www.ucl.ac.uk/slic) [6]. Schools were sampled by education performance within boroughs to ensure a wide range of socioeconomic circumstances. Anthropometry and spirometry (Easy-on-PC; ndd Medical, Zurich, Switzerland) were performed in schools according to international standards adapted for children [7,8] with follow-up assessments 12 months later. All assessments were undertaken by the same team of paediatric respiratory physiologists, using identical equipment and standardised protocols, with subsequent over-read by a senior respiratory physiologist to ensure appropriate quality control. Spirometry results were expressed as z-scores using the ethnic-specific 2012 Global Lungs Initiative equations [9], which adjust for sex, age, height, ethnicity, forced expiratory volume in $1 \mathrm{~s}$ (FEV1), forced vital capacity (FVC) and FEV1/FVC. Parents completed questionnaires about their child's ethnicity, birth data, and current and prior health status. Ethnicity was coded as White, Black (Black African or Black Caribbean), South Asian (Indian sub-continent) or other/ mixed. The study was approved by the London-Hampstead research ethics committee. Parents' written consent and children's verbal assent were obtained prior to assessments. Some results from this study have been reported previously $[10,11]$.

This study explores the impact of different exclusion criteria on mean spirometry results. Five exclusion criteria were considered. 1) Current or chronic lung disease, e.g. sickle cell disease, cystic fibrosis and current asthma (either doctor-diagnosed or asthma medication in the past 12 months, with or without current symptoms/wheeze). 2) Congenital abnormality likely to impact on lung development. 3) Born preterm (gestational age $<37$ weeks) or birthweight $<2.5 \mathrm{~kg}$. 4) Prior doctor-diagnosed asthma or hospitalisation for respiratory problems. 5) Symptomatic (cough or cold) at testing.

The first two exclusions were considered mandatory, while the impact of applying the remaining three was tested by comparing the overall results with and without them.

\section{Statistical analysis}

Results are presented as frequencies (\%) for categorical variables and mean $\pm \mathrm{SD}$ or median (range) for continuous outcomes. T-tests were used to compare mean differences in lung function between groups. The impact on the distribution of spirometry z-scores with different exclusion criteria was examined by comparing the mean and SD of the z-scores.

\section{Results}

Assessments were attempted in 2171 children on 3302 test occasions (including those from an initial feasibility study in two schools [6]). Of these, 125 children were excluded on technical grounds (124 who failed spirometry on all 279 test occasions, and one with missing height). A further 145 children (255 test occasions) were excluded under exclusion criteria 1 or 2: current or chronic lung disease, or congenital abnormality (table 1). Technically satisfactory spirometry was obtained for the remaining 1901 children on 2767 test occasions (46\% boys; 35\% White, 29\% Black, 24\% South Asian, 12\% other/mixed ethnicity; mean (range) age 8.3 (5.2-11.9) years). Technically acceptable spirometric data could not be obtained in $7.5 \%$ of all test occasions in healthy children. This failure rate was significantly higher among children with congenital abnormalities (mean difference (95\% CI) $18 \%(6.6-35 \%)$ ), current asthma (5.2\% $(2.5-8.5 \%))$, or those who were symptomatic at the time of the test $(26 \%(21-31 \%))$. 


\begin{tabular}{|c|c|c|c|c|}
\hline & Congenital abnormality\# & Sickle cell disease & Current asthma & Total \\
\hline Subjects $n$ & 9 & 12 & 124 & 145 \\
\hline Boys & 78 & 17 & 56 & 54 \\
\hline White & 56 & 17 & 32 & 32 \\
\hline Test occasions" $n$ & 8 & 18 & 192 & 218 \\
\hline Age at test years & $8.6 \pm 1.88$ & $8.4 \pm 1.83$ & $8.7 \pm 1.55$ & $8.7 \pm 1.58$ \\
\hline Height $^{+}$z-scores & $-0.17 \pm 1.87$ & $0.51 \pm 1.03$ & $0.27 \pm 1.29$ & $0.28 \pm 1.3$ \\
\hline FEV 1 z-scores & $0.19 \pm 2.68$ & $-0.50 \pm 1.01$ & $-0.27 \pm 1.04$ & $-0.27 \pm 1.13$ \\
\hline FVC z-scores & $0.27 \pm 2.82$ & $-0.37 \pm 1.00$ & $0.22 \pm 0.99$ & $0.17 \pm 1.10$ \\
\hline FEV $1 / F V C$ z-scores & $-0.05 \pm 0.90$ & $-0.29 \pm 1.04$ & $-0.85 \pm 0.96$ & $-0.77 \pm 0.98$ \\
\hline
\end{tabular}

Data presented are $\%$ or mean \pm SD, unless otherwise stated. $F E V_{1}$ : forced expiratory volume in $1 \mathrm{~s}$; FVC: forced vital capacity. " : congenital or neurological abnormalities, $n=6$; growth abnormalities, $n=3$; " : with technically acceptable spirometry results; ${ }^{+}$: defined according to [12].

Table 2 shows the 1901 children without chronic disease split into groups by identifying those meeting each of the exclusion criteria 3 to 5, while the remaining 1520 children constitute the healthy group. Among the children born preterm and/or low birthweight, the median (range) gestational age was 36 (23-41) weeks, with only five $(2.7 \%)$ being born before 28 weeks gestation representing $0.3 \%$ of the reference population. Similarly the mean (range) birthweight for this subgroup was $2.27(0.73-4.0) \mathrm{kg}$, with only three $(1.6 \%)$ children having a birthweight $<1 \mathrm{~kg}$. There was some overlap across the three exclusion groups, with $6-13 \%$ of children per group meeting more than one exclusion criterion. The proportions of children meeting the various criteria were similar across ethnic groups [6].

The mean \pm SD of the FEV1 and FVC $z$-scores approximated $0 \pm 1$ in the healthy group, indicating that the 2012 Global Lungs Initiative reference equations were broadly appropriate for this multi-ethnic population (table 2). Although there were no significant differences in FVC z-scores between the four groups, FEV1 $\mathrm{Z}$-scores and FEV1/FVC Z-scores were significantly lower in those with "prior asthma" or who were "symptomatic at test" by up to $0.3 \mathrm{z}$-scores for FEV1 (equating to $\sim 3.5 \%$ if expressed as \% predicted) and $0.5 \mathrm{z}$-scores for FEV1/FVC (table 2). Similar results were observed for forced expiratory flow at $25-75 \%$ of FVC but since it was no more discriminative in detecting children with lung function abnormalities than FEV1/FVC (data not shown) [13,14], this outcome was not reported for subsequent analyses.

\section{Impact of health status on lung function}

Relaxing the exclusion criteria to progressively include children born preterm and/or with low birthweight, children with prior asthma, and children symptomatic at testing had only minor effects on the z-score distributions of FEV1, FVC and FEV1/FVC for the entire group (table 3). Thus, despite the significant differences between the groups seen in table 2, the fact that the exclusion groups were relatively small

TABLE 2 Group characteristics and lung function according to health status ${ }^{\#}$ in 1901 children from 2767 test occasions

Healthy

Exclusion criterion

3: preterm/low birthweight" $\quad$ 4: prior asthma

5: symptomatic at test

\begin{tabular}{|c|c|c|c|c|}
\hline Subjects $n$ & 1520 & 186 & 158 & 111 \\
\hline Boys & 45 & 46 & 57 & 47 \\
\hline White & 37 & 24 & 33 & 38 \\
\hline Test occasions $^{+} \mathbf{n}$ & 2199 & 232 & 208 & 141 \\
\hline Height $^{\S}$ z-score & $0.48 \pm 1.05$ & $0.23 \pm 1.16$ & $0.41 \pm 1.14$ & $0.45 \pm 1.01$ \\
\hline FEV 1 z-score & $0.03 \pm 0.90$ & $-0.11 \pm 1.00$ & $-0.27 \pm 0.96^{* * *}$ & $-0.29 \pm 1.12^{* * *}$ \\
\hline FVC z-score & $0.17 \pm 0.92$ & $0.11 \pm 0.97$ & $0.12 \pm 0.87$ & $0.02 \pm 1.10$ \\
\hline
\end{tabular}

Data presented are \% or mean $\pm \mathrm{SD}$, unless otherwise stated. $\mathrm{FEV} 1$ : forced expiratory volume in $1 \mathrm{~s}$; FVC: forced vital capacity. ${ }^{\#}$ : exclusion criteria not mutually exclusive; ${ }^{\bigcap_{1}}:<2.5 \mathrm{~kg} ;{ }^{+}$: with technically acceptable spirometry results; ${ }^{\S}$ : defined according to [12]. ${ }^{* * *}:$ p<0.001 compared to lung function from healthy children. 
TABLE 3 Impact of health status on lung function

\begin{tabular}{|c|c|c|c|c|}
\hline & \multirow[t]{2}{*}{ Healthy } & \multicolumn{3}{|c|}{ Healthy plus } \\
\hline & & Preterm/low birthweight ${ }^{\#}$ & Prior asthma & Symptomatic at test \\
\hline Subjects $n$ & 1520 & 1676 & 1825 & 1901 \\
\hline Test occasions ${ }^{\pi} \mathbf{n}$ & 2199 & 2431 & 2626 & 2767 \\
\hline FEV1 z-score & $0.03 \pm 0.90$ & $0.02 \pm 0.90$ & $0.00 \pm 0.91$ & $-0.01 \pm 0.92$ \\
\hline FVC z-score & $0.17 \pm 0.92$ & $0.17 \pm 0.92$ & $0.17 \pm 0.92$ & $0.16 \pm 0.93$ \\
\hline
\end{tabular}

Data presented are \% or mean \pm SD, unless otherwise stated. FEV1: forced expiratory volume in $1 \mathrm{~s}$; FVC: forced vital capacity. ${ }^{\#}:<2.5 \mathrm{~kg} ;{ }^{~}$ : with technically acceptable spirometry results.

meant that including them with the healthy group made little difference to the combined $\mathrm{z}$-score distributions, but increased the reference sample size by 381 children or $25 \%$. Furthermore, the decrease of lung function among those with current asthma or chronic lung disease could still be distinguished from the reference sample (mean difference (95\% CI) (reference sample - current asthma): FEV1 z-score 0.26 (0.10-0.41); FEV1/FVC z-scores 0.53 (0.39-0.67)).

The 568 tests on the 381 unhealthy children constituted $20 \%$ of the total reference population. To explore how sensitive the conclusions were to this specific proportion, corresponding results were calculated by doubling the size of the unhealthy group, i.e. assuming a 60:40 split between the healthy and unhealthy test results rather than the observed 80:20. In practice this only had a small effect on the distribution, reducing the means for FEV $1 \mathrm{z}$-scores and FEV1/FVC $\mathrm{z}$-scores by 0.04 and 0.05 , respectively (no change was noticed for mean FVC z-scores), while increasing the SD by 0.01 , changes which happen to match the actual differences between the healthy and combined groups (table 3). Thus, the conclusions do not critically depend on the proportion of unhealthy children recruited from a large population sample, provided the sample is unselected and that there is no gross reduction in lung function among such children.

\section{Discussion}

Our study shows that, with the exception of children with clearly defined current or chronic disease, reference samples for paediatric spirometry can be relatively all-inclusive and thus more representative of the general population. While factors such as low birthweight, preterm delivery, prior asthma and symptoms at testing introduce bias in individuals, they do not have a substantial impact in large epidemiological studies due to the relatively small proportion of affected children, and the relatively mild reductions in lung function observed when recruiting an unselected population. Using this approach, the expanded sample in our study was not only more representative of the underlying population but also $25 \%$ larger, thereby increasing cost-effectiveness.

A major strength of our study is that all the assessments were undertaken by the same team using identical equipment and standardised protocols, with subsequent over-read by an experienced physiologist to ensure a high degree of quality control and reliability. As reported previously $[13,14]$, we found very little discordance between forced expiratory flow at 25-75\% of FVC and FEV1/FVC when classifying test results, suggesting forced expiratory flows do not contribute to clinical decision making in either children or adults. We recommend limiting the reporting of spirometry outcomes to FEV1, FVC and FEV1/FVC as recommended by the American Thoracic Society/European Respiratory Society guidelines [15].

This study was designed to assess children in school without parents needing to be present. This maximised recruitment and reduced bias that may have occurred had parents had to take time off work, wherein those with potential anxieties about their child's lung health may have been more willing to enrol. The proportions of preterm children and those with a diagnosis of asthma in the study were small, and similar to those reported for England and Wales (gestational age $<37$ weeks: $6 \%$; gestational age $<28$ weeks: 0.4\%; asthma: 9\%) [16, 17]. The study sample was also representative of an inner city population of multi-ethnic school children [18]. For the purposes of this study, any child born weighing $<2.5 \mathrm{~kg}$ or born at $<37$ weeks gestation was classified as low birthweight or preterm, respectively, but the vast majority of such children were relatively mature $(71 \%$ of this group were $\geqslant 35$ weeks gestational age and $67 \%$ were $\geqslant 2 \mathrm{~kg}$ birthweight), when any deficiencies in lung function are likely to be relatively minor [19].

The fact that neither prematurity nor low birthweight adversely affected the results in this unselected study, where such children represent only $8 \%$ of the population, does not diminish their potential impact 
in individual children, especially those born extremely preterm or of very low birthweight. This is clearly indicated by focussed studies (e.g. with a 50:50 mix of index cases and controls) where mean reductions in FEV1 by up to $1 \mathrm{z}$-score (i.e. $>10 \%$ ) have been reported $[4,5,20]$. Similarly, the need to record relevant prior medical history including birth status, and using such information when interpreting results, remains of paramount importance during both research studies and the clinical management of individual patients with respiratory disease at any age [21].

It was reassuring that current upper respiratory symptoms did not influence the sample distribution of spirometry, since not all epidemiological studies record symptoms during lung function testing [22], and such symptoms can be very subjective. However, it must be emphasised that these findings only apply to spirometry, which is expected to be relatively independent of upper respiratory symptoms. Furthermore, the failure rate was almost five times higher in those with symptoms than those without symptoms, suggesting a degree of "self-exclusion", with technically acceptable data being achievable only in children with relatively mild symptoms.

To assess the potential impact of including a higher proportion of unhealthy children on population estimates of spirometry, we modelled the effect of doubling the size of this group. Given that the proportions of children with prior asthma or those born preterm/low birthweight are unlikely to be higher than the unselected population sample from which they were recruited ( $15 \%$ of total), the effect of doubling the sample size of unhealthy children was just a crude approach to show that it makes little difference to the results, providing the mean deficit within such groups is minimal. The mean values fell slightly and the SD increased minimally, but in practice the impact was minimal, due both to the fact that the proportion of healthy children remained in the majority and that there were relatively small group differences in lung function between the healthy children and those with symptoms who were well enough to attend school and produce technically satisfactory results. It should be noted that since a $1 \mathrm{z}$-score change for FEV1 in 8 -year-old children is equivalent to $\sim 12 \%$ pred FEV1, a difference of $0.04 \mathrm{z}$-scores when doubling the proportion of unhealthy children only represents a change of $0.5 \%$ pred FEV1.

Our results suggest that where a genuinely healthy population sample of children is required to address a research hypothesis with spirometry as the primary outcome, i.e. where all five exclusion criteria apply, the target sample size needs to be increased by at least $30 \%$ to cover exclusions.

In conclusion, we found that the mean and SD of spirometry in our study was not materially affected by exclusion criteria such as mild current symptoms, prior wheeze or low birthweight. While inclusion/ exclusion criteria will always need to be considered carefully according to the specific hypotheses under examination, these findings have potential implications for epidemiological studies with respect to the cost, efficiency and generalisability of population studies with spirometric lung function as a primary outcome.

\section{Acknowledgements}

The Size and Lung function In Children study team acknowledges the support of the National Institute for Health Research (London, UK), through the Comprehensive Clinical Research Network. We thank Jane Kirkby (Great Ormond Street Hospital for Children NHS Foundation Trust, London, UK) and Emma Raywood, Sarah Legg, Dave Sears, Simon Lee and Philippa Cottam (all Respiratory, Critical Care and Anaesthesia Section, University College London, Institute of Child Health, London) for their help with data collection and data management. We would particularly like to thank the head teachers and staff of participating schools for facilitating the recruitment and school assessments, and last but not least the children and families who participated in the study.

\section{References}

1 Stanojevic S, Wade A, Stocks J. Reference values for lung function: past, present and future. Eur Respir J 2010; 36: $12-19$.

2 Muller-Brandes C, Kramer U, Gappa M, et al. LUNOKID: can numerical American Thoracic Society/European Respiratory Society quality criteria replace visual inspection of spirometry? Eur Respir J 2014; 43: 1347-1356.

3 Schwartz JD, Katz SA, Fegley RW, et al. Analysis of spirometric data from a national sample of healthy 6- to 24-year-olds (NHANES II). Am Rev Respir Dis 1988; 138: 1405-1414.

4 Stocks J, Sonnappa S. Early life influences on the development of chronic obstructive pulmonary disease. Ther Adv Respir Dis 2013; 7: 161-173.

5 Lum S, Kirkby J, Welsh L, et al. Nature and severity of lung function abnormalities in extremely pre-term children at 11 years of age. Eur Respir J 2011; 37: 1199-1207.

6 Lum S, Sonnappa S, Wade A, et al. Exploring ethnic differences in lung function: the size and lung function in children (SLIC) study protocol and feasibility. http://discovery.ucl.ac.uk/1417500/Date last updated: June 2014. Date last accessed: September 2014.

7 Kirkby J, Welsh L, Lum S, et al. The EPICure study: comparison of pediatric spirometry in community and laboratory settings. Pediatr Pulmonol 2008; 43: 1233-1241.

8 Miller MR, Hankinson J, Brusasco V, et al. Standardisation of spirometry. Eur Respir J 2005; 26: 319-338.

9 Quanjer PH, Stanojevic S, Cole TJ, et al. Multi-ethnic reference values for spirometry for the 3-95-yr age range: the global lung function 2012 equations. Eur Respir J 2012; 40: 1324-1343.

10 Lum S, Sonnappa S, Cole TJ, et al. How should we define a reference population when assessing lung function? Am J Respir Crit Care Med 2014; 189: A3229. 
11 Bonner R, Lum S, Stocks J, et al. Applicability of the global lung function spirometry equations in contemporary multiethnic children. Am J Respir Crit Care Med 2013; 188: 515-516.

12 Cole TJ, Freeman JV, Preece MA. British 1990 growth reference centiles for weight, height, body mass index and head circumference fitted by maximum penalized likelihood. Stat Med 1998; 17: 407-429.

13 Boutin B, Koskas M, Guillo $\mathrm{H}$, et al. Forced expiratory flows' contribution to lung function interpretation in schoolchildren. Eur Respir J 2015; 45: 107-115.

14 Quanjer PH, Weiner DJ, Pretto JJ, et al. Measurement of FEF25-75\% and FEF75\% does not contribute to clinical decision making. Eur Respir J 2014; 43: 1051-1058.

15 Pellegrino R, Viegi G, Brusasco V, et al. Interpretative strategies for lung function tests. Eur Respir J 2005; 26 : 948-968.

16 Moser K, Stanfield KM, Leon DA. Birthweight and gestational age by ethnic group, England and Wales 2005: introducing new data on births. Health Stat Q 2008; 39: 22-31.

17 Simpson CR, Sheikh A. Trends in the epidemiology of asthma in England: a national study of 333,294 patients. $J$ R Soc Med 2010; 103: 98-106.

18 Whitrow MJ, Harding S. Asthma in Black African, Black Caribbean and South Asian adolescents in the MRC DASH study: a cross sectional analysis. BMC Pediatr 2010; 10: 18.

19 Kotecha SJ, Watkins WJ, Paranjothy S, et al. Effect of late preterm birth on longitudinal lung spirometry in school age children and adolescents. Thorax 2012; 67: 54-61.

20 Hacking DF, Gibson AM, Robertson C, et al. Respiratory function at age 8-9 after extremely low birthweight or preterm birth in Victoria in 1997. Pediatr Pulmonol 2013; 48: 449-455.

21 Bolton CE, Bush A, Hurst JR, et al. Are early life factors considered when managing respiratory disease? A British Thoracic Society survey of current practice. Thorax 2012; 67: 1110.

22 Aresu M, Mindell J, Stocks J. Lung Function in Children. Health Survey for England - 2010: Respiratory Health. 2011. www.hscic.gov.uk/catalogue/PUB03023/heal-surv-eng-2010-resp-heal-ch5-func-chil.pdf Date last updated: December 15, 2011. Date last accessed: September 2014. 